\title{
PSICOPROFILAXIA CIRÚRGICA DE PACIENTES INFANTIS E ADOLESCENTES: PERCEPÇÃO DOS MÉDICOS
}

\author{
CHILD AND ADOLESCENT SURGICAL PSYCHOPROPHYLAXIS: PHYSICIANS' VIEW \\ PSICOPROFILAXIA QUIRÚRGICA DE NIÑOS Y ADOLESCENTES: PERCEPCIÓN DE LOS \\ MÉDICOS
}

\author{
Helena Bazanelli Prebianchi \\ Ariane Ferracini**
}

\begin{abstract}
RESUMO
O estudo teve por objetivo conhecer as percepções dos médicos cirurgiões, de um hospital-escola do interior paulista, sobre a psicoprofilaxia cirúrgica de pacientes infantis e adolescentes. Utilizou-se de entrevistas semiestruturadas com seis cirurgiôes pediátricos. As entrevistas seguiram um roteiro tópico flexível, e os dados coletados foram analisados mediante a análise de conteúdo temática de Bardin (2004). Os resultados indicaram que os participantes julgam necessária a preparação psicológica dos pais dos pacientes infantis para diminuir a ansiedade destes, torná-los mais cooperativos com a equipe de saúde e não influenciarem negativamente os comportamentos dos filhos. Contudo não adotam a psicoprofilaxia cirúrgica como prática rotineira, dirigindo-a apenas aos casos nos quais consideram que os comportamentos da criança ou dos pais prejudicam a recuperação e, ou, os procedimentos da equipe. Concluiu-se ser necessário aumentar a compreensão dos médicos, do hospital, sobre como as práticas de psicoprofilaxia cirúrgica podem satisfazer as necessidades da população atendida e aos profissionais envolvidos.
\end{abstract}

Palavras-chave: Psicoprofilaxia. Cirurgia. Criança.

\begin{abstract}
This study aims to learn the view surgeons, working for a school-hospital in the hinterland of São Paulo State, Brazil, have about the surgical psychoprophylaxis of child and adolescent patients. Six pediatric surgeons answered semi-structured Interviews. The interviews followed a flexible topic script and the resulting data were analyzed according to Bardin's method of content analysis (2004). The results indicated that the participants consider to be required the psychological preparation of pediatric patients' parents' in order to reduce their anxiety; make them more cooperative towards the health team and manage not to influence the children's behaviors in
\end{abstract}

Texto recebido em 21 de abril de 2016 e aprovado para publicação em 28 de junho de 2016.

·PUC Campinas.E-mail: helenabp@puc-campinas.edu.br.

**UC Campinas. E-mail: ariane.ferracini@hotmail.com. 
a negative way. However, they do not adopt surgical psychoprophylaxis as a routine practice, applying it only in cases where they consider that the child's or the parents' behavior affect the recovery and/or team procedures. As a conclusion, it is necessary to increase the physicians' understanding about how surgical psychoprophylaxis practices can meet the needs of the population and the professionals involved.

Keywords: Psychoprophylaxis. Surgery. Child.

\section{RESUMEN}

El objetivo del estudio fue conocer las percepciones de los médicos, de un hospital universitario, sobre la psicoprofilaxia quirúrgica de niños y adolescentes. Fueron realizadas entrevistas con seis cirujanos pediátricos, analizadas, posteriormente, según el método de análisis de contenido de Bardin (2004). Los resultados indicaron que los participantes consideran la necesidad de una preparación psicológica de los padres para disminuir la ansiedad de ellos y hacerlos más cooperativos con el equipo de salud y no influir negativamente en el comportamiento de los niños. Sin embargo, no adoptan la psicoprofilaxia quirúrgica como práctica habitual, y solo la utilizan en los casos en que consideran que el comportamiento del niño o de los padres pueden perjudicar la recuperación y los procedimientos del equipo. Se concluyó la necesidad de aumentar la comprensión de los médicos sobre cómo las prácticas de psicoprofilaxia quirúrgica pueden satisfacer las necesidades de la población atendida y de los profesionales implicados.

Palabras clave: Psicoprofilaxia. Quirugía. Niño.

\section{INTRODUÇÃO}

concepção da saúde, na perspectiva biopsicossocial, tem determinado que as práticas profissionais, nessa área, realizem-se com base na articulação 1 Lentre saberes de diferentes campos do conhecimento, isto é, cada vez mais, as ciências são chamadas à interação e interdependência.

Nesse sentido, o atendimento à saúde do paciente cirúrgico, no contexto hospitalar, é um campo de atuação desafiador a todas as áreas do conhecimento envolvidas nessa assistência, uma vez que uma série de procedimentos executados por diferentes profissionais ocorre concomitantemente e deve compor um conjunto integrado de cuidados ao processo cirúrgico (Turra, Costa Junior, Almeida, \& Doca, 2011).

Segundo Garretson (2004), a hospitalização isolada causa estresse, e este é ainda maior em pacientes cirúrgicos. $\mathrm{O}$ estresse surge da transação pessoa- 
ambiente e, por isso, as abordagens psicológicas do estresse têm enfatizado o papel dos processos cognitivos de avaliação e expectativa, isto é, o estresse está relacionado à avaliação do indivíduo do grau de ameaça numa situação em relação aos recursos ambientais disponíveis e às capacidades de enfrentamento individuais (Mullins et al., 2007). Para Broering e Crepaldi (2011), a cirurgia é um estressor psicossocial, visto que os fatores pessoais do paciente, tais como estrutura psíquica, cultura, relaçôes familiares, o conhecimento ou não, sobre o procedimento, bem como procedimentos bem-sucedidos ou não, realizados anteriormente, determinam sua interpretação.

Em estudo de revisão da literatura, sobre o tema, Turra et al. (2011) encontraram que os estressores mais significativos dentro da situação cirúrgica são o diagnóstico, a doença, a dor, a hospitalização, os procedimentos médicos, o temor de não despertar da anestesia, as consequências da cirurgia, a perda $\mathrm{da}$ autonomia e a morte. Esses fatores ansiogênicos podem interferir, de modo adverso, sobre a aquisição de estratégias de enfrentamento do procedimento cirúrgico e sobre o processo de recuperação do paciente, com maior probabilidade, então, de episódios de elevação da pressão sanguínea, sangramentos mais intensos nas cirurgias, redução de resistência imunológica e transtornos psicossomáticos (Ribeiro, Tavano, \& Neme, 2002).

Especificamente em relação aos pacientes infantis, alguns autores indicam que a hospitalização e a cirurgia são estressantes e produzem experiências de ansiedade que podem desencadear distúrbios psicológicos na maioria das crianças, num período transitório ou a longo prazo (Broering \& Crepaldi, 2011; Barros, 1999). Segundo Lipp e Lucarelli (2005), o estresse infantil assemelha-se ao do adulto em vários aspectos e pode gerar sérias consequências no caso de ser excessivo.

O impacto da hospitalização sobre o comportamento da criança leva, na maioria das vezes, à manifestação de reaçóes adversas, como estresse, ansiedade e medo (Salmon, 2006; Broering \& Crepaldi, 2011). Schmitz, Piccoli, e Vieira (2003) afirmam que a criança internada para realização de procedimento cirúrgico sai de sua rotina e, devido ao medo do desconhecido, está suscetível a distúrbios psicológicos, advindos do desconforto, ansiedade e alterações do sensório, tais como turvação da consciência, alucinaçóes, ilusões e desorientação (Vaughan, \& McKay, 1977 apud Schmitz, Piccoli, \& Vieira, 2003, p. 67).

De forma geral, a atividade do psicólogo hospitalar na atenção ao paciente infantil objetiva minimizar o sofrimento das crianças naquele contexto e favorecer um ambiente menos hostil, independentemente do tempo e da doença que as levaram à internação (Mesquita, Silva, \& Rocha Júnior, 2013). A preparação psicológica é caracterizada por Quiles e Carrillo (2000) como um 
conjunto de procedimentos técnicos que tem por objetivo geral aliviar as reaçôes emocionais advindas da situação de internação hospitalar e facilitar a adaptação comportamental a esse contexto.

Costa Junior et al. (2012) afirmam que a preparação psicológica possibilita ressignificar o estado atual do paciente, melhorar os recursos psíquicos para enfrentamento da situação estressora e generalizar para os diversos momentos do tratamento. Segundo os autores, essa preparação consiste em amenizar o sofrimento do paciente, torná-lo participativo, possibilitar a expressar livremente seus sentimentos e preocupações, auxiliá-lo a controlar sua ansiedade, facilitar a canalização de informações necessárias, adequadas e suficientes, avaliar suas condições psicoemocionais.

Em razão do momento de ocorrência, a preparação pode acontecer em três fases distintas (Doca \& Costa Júnior, 2007):

a) antes da hospitalização;

b) no momento da admissão hospitalar; ou

c) antes de exames e procedimentos médicos invasivos, tais como punções e cirurgias.

Os programas de preparação psicológica executados antes da internação constituem ações preventivas, de cunho educativo, dirigidos a crianças sem experiência de hospitalização, e podem ser desenvolvidos tanto na comunidade quanto em ambulatório. Os programas de preparação desenvolvidos no momento da admissão hospitalar mantêm o caráter preventivo dos anteriores e envolvem o fornecimento de informações sobre normas e rotinas da unidade, os procedimentos que serão executados e o treino em habilidades de enfrentamento. Em ordem decrescente, as formas mais comuns de repasse dessas informaçóes são:

a) oral, durante os atendimentos ao paciente;

b) impressa, por meio de folhetos e cartilhas; e

c) audiovisual, por meio da projeção de slides e filmes de curta duração (Barros, 1999; Crawford, 2002; Scott \& Arslanian-Engoren, 2005).

De fato, estudos têm mostrado que programas de preparação psicológica de crianças para cirurgia reduzem sua ansiedade, medos, angústias e preocupações 
(Magalhães, Gusmam, \& Grecca, 2010; Carvalho, 2012; Gabarra \& Crepaldi, 2011b), permitem a elaboração de suas fantasias (Peixoto \& Mazambani, 2008), aumentando sua calma e qualidade de sono (Gabarra \& Crepaldi, 2011b). No entanto se verifica também que a comunicação familiar, muitas vezes, é a principal fonte de informação para as crianças sobre a doença e que profissionais e a família tendem a omitir informações com o intuito de protegê-las de maiores sofrimentos (Gabarra \& Crepaldi, 2011a).

Assim, este estudo objetiva conhecer as percepções dos médicos cirurgiões de um hospital universitário sobre a psicoprofilaxia cirúrgica de pacientes infantis e adolescentes.

\section{MÉTODO}

\subsection{Participantes}

Os participantes foram seis médicos ( 4 homens e 2 mulheres) com experiência profissional de 3 a 22 anos, e idade entre 30 e 48 anos. Os médicos compóem a equipe de cirurgia pediátrica de um hospital universitário de uma cidade do interior paulista, o qual conta com 353 leitos ativos, sendo 243 destinados exclusivamente ao convênio do Sistema Único de Saúde (SUS).

\subsection{Instrumentos}

Entrevista semiestruturada composta por questôes norteadoras que buscaram alcançar os objetivos propostos pelo estudo a saber:

a) o que você entende por psicoprofilaxia cirúrgica?;

b) na sua opinião, qual a importância da psicoprofilaxia cirúrgica?;

c) com que frequência seus pacientes são submetidos a psicoprofilaxia cirúrgica?

\subsection{Procedimentos de coleta de dados e éticos}

A coleta ocorreu de forma individual nos locais de trabalho dos participantes. A pesquisa foi desenvolvida de acordo com as normas preconizadas pela Resolução 196/96 do Conselho Nacional de Saúde, que trata de pesquisas envolvendo seres humanos. Desse modo, a pesquisa foi aprovada pelo Comitê de Ética da 
instituição, sob o número CEP 410.662. O convite para participação na pesquisa foi realizado pessoalmente pela primeira autora.

No início de cada um dos encontros, a pesquisadora apresentava ao participante o termo de consentimento livre e esclarecido para ser lido e assinado. Em seguida, iniciava a entrevista com os aspectos definidos pelo roteiro. As entrevistas foram gravadas por meio de um aparelho de mídia player e, posteriormente, transcritas. O material empírico foi coletado no período de outubro de 2014 a março de 2015, em horários e locais previamente acordados com os entrevistados.

As transcrições foram submetidas à análise de conteúdo do tipo categorial temática, proposta por Bardin (2004). Especificamente, para a análise, procederam-se os seguintes passos:

a) transcrição das entrevistas;

b) demarcação das unidades de sentido; e

c) geração de categorias temáticas, sendo estas criadas com base nos relatos dos médicos.

\section{RESULTADOS E DISCUSSÃO}

Com base no discurso dos participantes, foi identificado um grande eixo temático que, por sua vez, deu origem às seguintes categorias e subcategorias (quadro 1).

Quadro 1 - Categorias e subcategorias do eixo temático

\begin{tabular}{|c|c|c|}
\hline Tema & Categorias & Subcategorias \\
\hline \multirow{2}{*}{ Psicoprofilaxia } & O que é & Desconhecimento \\
& & Preparação da família \\
& & Feita por médicos \\
\cline { 2 - 3 } & & Feita por psicólogos em casos especiais \\
& Importância & Redução da ansiedade \\
& & Prevenir complicações \\
& & Satisfação do paciente \\
& & Prevenir influência negativa dos pais no pós-operatório \\
\hline
\end{tabular}

Fonte: elaborado pelas autoras. 


\subsection{Eixo temático: psicoprofilaxia cirúrgica}

Aborda as dimensões de definição ou conceituação da psicoprofilaxia cirúrgica ou o desconhecimento sobre esta, como tem sido realizada e sua importância em relação aos aspectos: redução da ansiedade dos pacientes e familiares, adesão ao tratamento, prevenção de complicações, satisfação do paciente e prevenção de influência negativa dos pais no pós-operatório.

\subsection{O que é: desconhecimento; feita por médico; feita por psicólogo em casos especiais}

Uma primeira condição para o trabalho multidisciplinar efetivo é a clareza das atribuiçôes de cada profissional da equipe e das expectativas concernentes a cada especificidade. $\mathrm{O}$ trabalho multiprofissional, na lógica da interdisciplinaridade, é uma possibilidade de ampliar a capacidade humana de compreender a realidade e os problemas que nela se apresentam. O multiprofissionalismo se refere à recomposição de diferentes processos, que devem flexibilizar a divisão do trabalho; preservar as diferenças técnicas entre os trabalhadores especializados; diminuir as desigualdades na valorização dos distintos trabalhos e respectivos agentes, bem como nos processos decisórios, e compreender a interdependência dos saberes para a execução e cumprimento do mesmo objetivo (Oliveira et al., 2011).

Os depoimentos de alguns participantes, contudo, denotam a dificuldade de envolver diferentes profissionais que estejam preparados para intercambiar saberes de forma que se complementem, gerando alternativas e soluções pertinentes e eficazes para os casos abordados: "Não sei do que se trata. O que é psicoprofilaxia cirúrgica?" (Médico 1); "Não sei. O que trata a psicoprofilaxia cirúrgica?" (Médico 4). Estudos têm mostrado que os médicos têm uma falta real de conhecimento acerca da Psicologia, relacionando-a a uma função assistencialista, fortemente associada à psicologia clínica, sendo que tal defasagem provém da própria formação médica e se estende na atuação, visto que não se encontra, nos hospitais, a psicologia hospitalar como uma área delimitada e de profissionais com papéis bem definidos (Gazotti \& Prebianchi, 2014; Barreto \& Castro, 2015).

Por outro lado, o aumento no número de profissionais da Psicologia no contexto hospitalar não foi acompanhado por um crescimento compatível na quantidade de pesquisas e nas diversas possibilidades de formação qualificada (Gazotti \& Prebianchi, 2014, Barreto \& Castro, 2015). Como indicado por AngeramiCamom (2010), a Psicologia hospitalar, como área de atuação da Psicologia, 
tem assumido um modelo próprio de atuação, adaptado à realidade institucional dos hospitais, a fim de atender às necessidades de pacientes, familiares e equipe. Nesse sentido, o estudo realizado confirma que uma das atividades do psicólogo hospitalar, principalmente nos hospitais-escolas, é a interconsulta (Gazotti \& Prebianchi, 2014; Rossi, 2008), conforme as declaraçoes: "Quando existe algum caso específico, a gente solicita a Psicologia [. . .]" (Médico 2); "Se for uma paciente que você nota que tem risco, aí você pode solicitar a psicóloga" (Médico 5); "Então assim, dependendo do caso (referindo-se a uma criança submetida a várias cirurgias), acontece uma intervenção psicológica que é solicitada pela equipe" (Médico 4).

O despreparo dos profissionais da saúde para a interdisciplinaridade, aliás, não pode ser creditado apenas à sua formação, mas também às condições para o exercício profissional, ou seja, considerada a perspectiva das definições políticas mais amplas, a baixa prioridade dada pelo governo brasileiro à questão da saúde tem impacto negativo sobre a estrutura, organização e funcionamento das instituiçôes a ela relacionadas. Nas palavras dos participantes, isso parece evidente:

"Então, isso é feito pelo próprio cirurgião e pelo anestesista na consulta pré-anestésica. O psicólogo não está na consulta pré-operatória. Porque assim, o paciente tem duas consultas: no pré, uma consulta inicial para avaliar o problema e solicitar a avaliação anestésica e na segunda, ele vem com resultado de exame e liberação anestésica. E a partir disso já é marcada a cirurgia" (Médico 5).

"Hoje em dia cabe ao médico fazer isso, então ele fica sobrecarregado porque ele tem que fazer muitas funçôes que não é dele” (Médico 6).

"Nós também fazemos isso de uma forma mais leiga, de forma empática, a partir do momento em que você discute com seu paciente sobre as implicaçôes" (Médico 1).

"Da preparação psicológica eu entendo que nós médicos fazemos alguma coisa dentro do consultório, conversando e esclarecendo" (Médico 4).

\subsection{O que é: preparação da família}

Considerando-se que os pais exercem influência significativa no comportamento dos filhos, eles também requerem orientação e preparação para a hospitalização das crianças (Broering \& Crepaldi, 2011).

No Brasil, alguns esforços, em termos de políticas públicas, têm sido realizados para tornar mais amenos os impactos decorrentes da internação da criança, como 
o Estatuto da Criança e do Adolescente (Lei no 8.069, 1990) e a Resolução no 41/1995, que garantem que acompanhantes de crianças e adolescentes participem ativamente do diagnóstico, tratamento e prognóstico ao receber informações sobre os procedimentos aos quais seus filhos serão submetidos.

Dessa forma, ocorreu uma mudança nos objetivos e processos de cuidados: agora, a intervenção é centrada na família e, em termos de processo, o modelo prevalente é uma relação pais-profissional colaborativa que apoia os pais em atingirem seus objetivos e os capacita, ajudando-os a adquirir o conhecimento e as habilidades necessárias para atender às necessidades especiais de seus filhos. Os participantes confirmam essa perspectiva:

"Eu entendo que é um serviço que auxilia o paciente a diminuir o grau de ansiedade. E no nosso caso de paciente infantil, muitas vezes a ansiedade da criança é proveniente da ansiedade dos pais. Então esse processo ajuda nisso" (Médico 2).

"Eu entendo que é uma forma de preparar não só o paciente mais como os pais no processo cirúrgico" (Médico 3).

"Eu entendo que éuma orientação as mães, explicando o procedimento anestésico e o procedimento cirúrgico" (Médico 5).

"Eu entendo que é você preparar o ambiente familiar da criança, pra ela que vai entrar numa sala sem a mãe pra fazer um processo cirúrgico, que é necessário. As crianças pequenas são muito mais fáceis operar. Agora os pais sofrem muito mais que as crianças, geralmente" (Médico 6).

Os resultados permitem, todavia, conjecturar que, além da mudança nos processos de cuidados, outros aspectos condicionam os depoimentos dos participantes. Suas afirmações parecem traduzir o não reconhecimento da relevância dos processos psicológicos (comportamentais, cognitivos e emocionais) para os problemas da saúde infantil ou que problemas de comportamento infantil podem resultar da interação de processos físicos e psicológicos:

"As crianças são ingênuas e inocentes, então esse processo passa totalmente desapercebido para elas. Os mais velhos podem até futuramente lembrar, mas as mais novinhas" (Médico 3).

"Eu acho que o que precede sempre é o mais importante, sempre tentando aliviar essa angústia, principalmente por parte dos pais, porque, para as crianças, esse processo (psicoprofilaxia) é irrelevante e visto que as explicaçôes são feitas diretamente com os pais, então eles que acabam lidando com isso" (Médico 3). 
"Eu acho mais importante preparar a mãe do que o filho [. . .] Eu tento brincar e ir conversando com a criança e isso faz com ela vá com sua cara" (Médico 6).

"Então a psicoprofilaxia tem de reforçar a indicação. E não dar a autonomia pro cliente" (Médico 1).

Além disso, essas afirmações são consonantes com achados como os de Gabarra e Crepaldi (2011a), numa pesquisa que visou a descrever a comunicação médicopaciente pediátrico-família na perspectiva da criança: as crianças referiram que os profissionais conversam mais com os seus pais do que com elas, e a comunicação familiar foi indicada como a principal fonte de informação sobre a doença, evidenciando, assim, que, embora as crianças possuam capacidade de compreender e perceber o que ocorre com o seu corpo, os profissionais e a família tendem a omitir informações.

A preparação para a hospitalização e cirurgia é um direito da criança como doente, cabendo aos profissionais envolvidos uma postura afetiva e disponível, atendendo a suas necessidades e direitos, especialmente no que diz respeito à informação antecipada sobre todos os procedimentos a serem realizados. Nesse sentido, a Psicologia pediátrica tem contribuído substancialmente com estudos sobre adaptação à doença, procedimentos médicos invasivos e hospitalização, promoção da saúde e o trabalho com os pais (Carvalho, 2012; Grilo \& Pedro, 2005; Barros, 1999), mas, infelizmente, esses conhecimentos não parecem ser empregados pelos profissionais médicos e psicólogos no hospital onde ocorreu esta pesquisa.

Pelo contrário, os resultados evidenciam que, a despeito do tempo de atuação no hospital, contexto deste estudo, todos os participantes desconhecem qualquer tipo de programa de preparação psicológica, e apenas alguns deles têm experiência com a solicitação dessa atividade, em casos específicos. Questiona-se em que medida também os psicólogos que ali trabalham contribuem para a manutenção desse desconhecimento, por meio da limitação imposta institucionalmente a suas funçôes ou, conforme estudo de Turra et al. (2011), devido à sua própria ausência de consenso teórico ou metodológico no tocante à nomenclatura de suas atividades, aos objetivos, às técnicas e aos procedimentos de execução.

\subsection{Importância: redução da ansiedade e prevenir complicações}

O estresse e a ansiedade pré-cirúrgica aumentam o período de recuperação. São obstáculos contraproducentes na reabilitação do paciente. Foi demonstrado que quanto maior o nível de estresse de um paciente antes da cirurgia, maior é a 
demora do processo de cicatrização, maior a debilidade do sistema imunológico e pode mostrar-se pouco colaborador com os procedimentos médicos (Söderman, Lisspers, \& Sundin, 2007). Os participantes indicam o reconhecimento desses aspectos ao falarem sobre a importância da psicoprofilaxia cirúrgica:

"É importante para tranquilizar a criança da situação cirúrgica, do processo da internação" (Médico 2).

"Exatamente isso, diminuir essa ansiedade dos pais em relação ao procedimento cirúrgico dos filhos [...] Esse equilíbrio bioquímico no nosso organismo que vai ser alterado em função do estresse. Então evitar isso num procedimento cirúrgico é um ponto positivo" (Médico 3).

"As pessoas esquecem do individuo, do ser humano e principalmente, no caso de criança, em que a ansiedade é muito maior e a preocupação da família" (Médico 4).

"Se você fizer um bom pré-operatório, o seu pós-operatório é bem mais tranquilo. E se você tiver algum tipo de complicação, foi conversado tudo antes. Os profissionais que não fazem uma preparação no pré-operatório têm muito mais complicação no pós operatório" (Médico 6).

"O que melhora muito na comunicação dos psicólogos com os pacientes é no entendimento de que a complicação não significa erro" (Médico 1).

\subsection{Importância: satisfação do paciente}

A partir da criação do SUS, tem-se objetivado a reformulação dos serviços de atenção à saúde, desde a formação dos profissionais envolvidos até a ampla reforma dos modelos e reorganização dos recursos materiais e humanos, sob a égide da interdisciplinaridade, intersetorialidade, embasadas numa leitura biopsicossocial. Simultaneamente, o processo de implantação da qualidade nos hospitais tem impulsionado a mensuração do constructo "satisfação do usuário", como parte integrante das avaliaçôes de resultado que, por sua vez, norteiam as decisões dos gestores de saúde.

No caso do hospital universitário, contexto desta pesquisa, o processo de sua creditação pelo Ministério da Saúde é uma realidade refletida nas palavras do Médico 4: "A importância é o paciente sair satisfeito [. . .] Tentar tirar do paciente esse fator da surpresa e deixá-lo esclarecido sobre o tratamento. Essa é a importância da psicoprofilaxia”. 


\subsection{Importância: prevenir influência negativa dos pais no pós-operatório}

Os comportamentos dos pais influenciam as reações emocionais da criança e sua percepção da experiência de hospitalização (Barros, 1999; Crawford, 2002), indicando que eles podem ser elementos-chave no processo de adaptação e enfrentamento, ao oferecerem modelos funcionais e adequados, ao estimularem o desenvolvimento dessas habilidades em seus filhos e ao oferecer-lhes apoio emocional incondicional. No entanto, para que isso ocorra, pode ser necessário disponibilizar treinamento e suporte emocional especializado aos pais e acompanhantes (Costa Junior, 2004), com o que concordam os participantes:

"E principalmente na utilização dessa preparação com a família. Explicar para essa família, para essa mäe, como seu filho vai voltar da cirurgia [. . . Porque, muitas vezes, nós explicamos, e mãe fala que entendeu, mas, quando a criança chega no pós-operatório, a mãe fica surpresa com o resultado, porque ela não tinha entendido nossa explicação e orientação do processo cirúrgico" (Médico 2).

"A importância é deixar a família mais tranquila e, com isso, participar mais do pós-operatório" (Médico 5).

Quando o paciente internado é uma criança, as perdas e os fatores de estresse afetam mais diretamente seus familiares. Estes, por sua vez, podem afetar o estado emocional da criança e seu repertório de comportamentos diante da doença e do tratamento, especialmente quando o adulto é a mãe, o pai ou pessoa de referência pessoal da criança (Costa Junior, 2004; Doca \& Costa Júnior, 2007). Como Barros (2010), o Médico 3 acredita que um adulto calmo e seguro ajuda a criança a organizar-se e confiar; pais tensos e ansiosos, e profissionais pouco preparados e igualmente ansiosos contribuem para aumentar a ansiedade da criança:

"Porque você vai estar passando uma tranquilidade, lidando com essa angústia e sofrimento dos pais, não deixando isso extravasar para a criança. Uma vez que você consegue controlar isso com os pais, você consegue controlar isso com a criança [.. . Então, se eu lidar de forma positiva com os pais, consequentemente, eu consigo lidar de forma positiva com as crianças" (Médico 3).

\section{CONCLUSÃO}

Pelas falas dos entrevistados é possível concluir que, no hospital contexto desta pesquisa, as dificuldades na atuação interdisciplinar têm se constituído um obstáculo à efetivação da psicoprofilaxia cirúrgica de pacientes infantis.

Ainda que os médicos reconheçam a necessidade de preparação psicológica dos pais dos pacientes infantis como uma estratégia para diminuir a ansiedade 
destes e torná-los mais cooperativos com a equipe de saúde e não influenciarem negativamente os comportamentos dos filhos durante a internação, eles não adotam a psicoprofilaxia cirúrgica como prática rotineira e sistemática. A preparação psicológica para a cirurgia é dirigida apenas aos casos nos quais os médicos entendem que o comportamento e, ou, reações emocionais da criança ou dos pais prejudicam a recuperação e, ou, os procedimentos da equipe.

Dessa forma, os médicos não atribuem legitimidade ao discurso especializado acerca da profilaxia cirúrgica para pacientes infantis, dificultando a adoção sistemática, no cotidiano hospitalar, da preparação psicológica para a cirurgia de crianças.

A literatura da área reconhece que o fornecimento de informação objetiva e concreta sobre situações estressantes facilita o confronto com estas, devido à formação de esquemas cognitivos que permitem aumentar o grau de previsibilidade, diminuir o de discrepância entre o esperado e o vivido e aumentar a capacidade da criança/adolescente para compreender e interpretar a experiência. Assim, a implementação de programas de preparação para a hospitalização é necessária para evitar manifestações de ansiedade e problemas de comportamento durante e após a internação e a cirurgia, e para prevenir outro tipo de perturbações no desenvolvimento infantil ou no relacionamento familiar a médio prazo.

Nesse sentido, conclui-se ser necessário aumentar a compreensão dos médicos sobre como as práticas de psicoprofilaxia cirúrgica, no hospital em questão, podem ser capazes de levar em consideração os desejos e necessidades da população atendida e satisfazer aos profissionais envolvidos. Para tanto, é imprescindível que a instituição de saúde reveja sua organização e funcionamento no que tange às cirurgias de pacientes infantis bem como que os psicólogos que ali trabalham façam uma reflexão sobre o seu modelo de atuação e sua inserção na equipe.

E, finalmente, constatada a escassez de pesquisas que investiguem a experiência dos cirurgiōes com a preparação psicológica de seus pacientes e tendo em vista a importância de sua avaliação e conhecimento sobre o tema, recomenda-se a realização de novos estudos. 


\section{REFERÊNCIAS}

Angerami-Camom, V. A. (2010). O psicólogo no hospital. In V. A. AngeramiCamom, Psicologia hospitalar: teoria e prática. (pp. 1-14). São Paulo: Cengage Learning.

Bardin, L. (2004). Análise de conteúdo (3a ed.). Lisboa: Editora 70.

Barreto, S. M. \& Castro, E. K. (2015). Critérios de médicos oncologistas para encaminhamentos psicológicos em cuidados paliativos. Psicologia: Ciência e Profissão, 35(1), 69-82.

Barros, L. (1999). Psicologia pediátrica: perspectiva desenvolvimentista. Lisboa: Climepsi.

Barros, L. (2010). A dor pediátrica associada a procedimentos médicos: contributos da psicologia pediátrica. Temas em Psicologia, 18(20), 295-306.

Broering, C. V. \& Crepaldi, M. A. (2011). Preparação psicológica e o estresse de crianças submetidas a cirurgia. Psicologia em Estudo, 16(1), 15-23.

Carvalho, M. D. R. (2012). Efeitos de um programa de preparação psicológica para o internamento na redução dos niveis de ansiedade em adolescentes hospitalizados (Dissertação de Mestrado). Universidade de Coimbra, Faculdade de Psicologia e Ciências da Educação, Coimbra.

Costa Junior, A. L. (2004). A intervenção psicológica no contexto de procedimentos médicos invasivos em oncologia pediátrica: discussão metodológica. In M. Z. S. Brandão (Org.), Sobre comportamento e cognição. (pp. 139-151). Santo André: Esetec.

Costa Junior, A. L., Doca, F. N. P., Araújo, I., Martins, L., Mundim, L., \& Sidrim, A.C. (2012). Preparação psicológica de pacientes submetidos a procedimentos cirúrgicos. Estudos de Psicologia, 29(2), 271-284.

Crawford, D. A. (2002). Keep the focus on the family. Journalof Child Health Care, 6, 133-146.

Doca, F. N. P. \& Costa Junior, A. L. (2007). Preparação psicológica para admissão hospitalar de crianças: uma breve revisão. Paidéia, 17(37), 167-179.

Gabarra, L. M. \& Crepaldi, M. A. (2011a). A comunicação médico-paciente pediátrico-família na perspectiva da criança. Psicologia Argumento, 29(65), 209-218. 
Gabarra, L. M. \& Crepaldi, M. A. (2011b). Percepções das mães sobre a preparação pré-cirúrgica de seus filhos segundo dois modelos. Psicologia Argumento, 29(66), 327-341.

Garretson, S. (2004). Benefits of pre-operative information programmes. Nursing Standart, 18(47), 33-37.

Gazotti, T. C. \& Prebianchi, H. B. (2014). Caracterização da interconsulta psicológica em um hospital geral. Psicologia: Teoria e Prática, 16(1), 18-30.

Grilo, A. M. \& Pedro, H. (2005). Contributos da Psicologia para as profissões da saúde. Psicologia, Saúde \& Doenças, 6(1), 69-89.

Lei no 8.069 de 13 de julho de 1990. (1990, 13 julho). Dispõe sobre o Estatuto da Criança e do Adolescente e dá outras providências. Brasília: Senado federal.

Lipp, M. E. N. \& Lucarelli, M. D. M. (2005). Escala de stress infantil: ESI: manual. São Paulo: Casa do Psicólogo.

Magalhães, F. M., Gusmam, D. P. P., \& Grecca, K. R. (2010). Preparo psicológico em pacientes submetidos a cirurgia cardíaca pediátrica. Revista Brasileira de Terapias Cognitivas, 6(2), 142-161.

Mesquita, D. A., Silva, E. P., \& Rocha Junior, J. R. (2013). O psicólogo atuando junto àcriança hospitalizada. Cadernos de Graduação - Ciências Biológicas e da Saúde Fits, 1(2), 89-96.

Mullins, L. L., Wolfe-Christensen, C., Pai, A. L. H., Carpentier, M. Y., Gillaspy, S., Cheek, J., \& Page, M. (2007). The relationship of parental overprotection, perceived child vulnerability, and parenting stress to uncertainty in youth with chronic illness. Journal of Pediatric Psychology, 32(8), 973-982.

Oliveira, E. R. A., Fiorin, B. H., Lopes, I. J., Gomes, M. J., Coelho, S. O., \& Morra, J. S. (2011). Interdisciplinaridade, trabalho em equipe e multiprofissionalismo: concepções dos acadêmicos de enfermagem. Revista Brasileira de Pesquisa em Saúde, 13(4), 28-34.

Peixoto, E. A. \& Mazambini, A. (2008). Programa de preparo psicológico préoperatório infantil. In Anais do 1 Encontro Regional de Psicologia, Maringá. Maringá: Cesumar.

Quiles, J. M. O., \& Carrillo, F.X. M. (2000). Hospitalización infantil:repercusiones psicológicas: teoria y práctica. Madrid: Biblioteca Nueva. 
Resolução no 41, de 17 de outubro de 1995. (1995, 17 outubro). Dispõe sobre os direitos da criança hospitalizada. Conselho Nacional de Defesa dos Direitos da Criança e Adolescente. Diário Oficial da República Federativa do Brasil, seção I, 16319-16320.

Resolução no 196, de 10 de outubro de 1996. (1996, 10 outubro). Diretrizes e normas regulamentadoras de pesquisas envolvendo seres humanos. Brasília: Conselho Nacional de Saúde. Recuperado a partir de http://www.scielo.br/ pdf/jpneu/v25n2/v25n2a6.pdf

Ribeiro, R. M., Tavano, L. D. A., \& Neme, C. M. B. (2002). Intervenções psicológicas nos períodos pré e pós-operatório com pacientes submetidos a cirurgia de enxerto ósseo. Estudos de Psicologia, 19(3), 67-76.

Rossi, L. (2008). Gritos e sussurros: a interconsulta psicológica nas unidades de emergências médicas do Instituto Central do Hospital das Clínicas - FMUSP. (Dissertação de Mestrado). Instituto de Psicologia da Universidade de São Paulo, Programa de Pós-Graduação em Psicologia Clínica, São Paulo.

Salmon, K. (2006). Preparing young children for medical procedures: taking account of memory. Journal of Pediatric Psychology, 31(8), 859-861.

Schmitz, M. S., Piccoli, M., \& Vieira, C. S. (2003). A criança hospitalizada, a cirurgia e o brinquedo terapêutico: uma reflexão para a enfermagem. Ciência, Cuidado e Saúde, 2(1), 67-73.

Scott, L. D., \& Arslanian-Engoren, C. (2005). The decision to care: a lifealtering experience. Home Health Care Management \& Practice, 17, 130-135.

Soderman, E., Lisspers, J., \& Sundin, O. (2007). Impact of depressive mood on lifestyle changes in patients with coronary artery disease. J Rehabil Med., 39(5), 412-417.

Turra, V., Costa Junior, A. L., Almeida, F. F., \& Doca, F. N. P. (2011). Contribuiçôes da Psicologia na atenção ao paciente cirúrgico: uma análise da literatura. Comunicação em Ciências da Saúde, 22(4), 353-366. 